\title{
Application of Machine Learning Methods to Interpolation of Aircraft Aerodynamic Data
}

\author{
Myeong-Jae Jo, Won-Hyuck Choi and Min-Seok Jie* \\ Dept. of Aeronautical System Engineering, Hanseo University, Taean, \\ Republic of Korea \\ legnahels@naver.com,choiwh@hanseo.ac.kr,jiems@hanseo.ac.kr
}

\begin{abstract}
The collection and processing of aerodynamic data to estimate aircraft speed or attitude is an essential process in flight. In this paper, machine learning methods were applied for aerodynamic data processing and optimization of collected aircraft aerodynamic data. The proposed method is a 'supervised learning' among the methods of machine learning, in which artificial neural networks are learned based on the accumulated data. For this purpose, input and output of accumulated data must be strictly defined for train of neural network, and the original output value of the input is compared with the estimated value of the learned neural network. What the proponents used as neural network structure was multilayer perceptron (MLP, Multi-Layer Perceptron), which consists of two layers for hidden layers excluding the input layer and the output layer for estimates the result using linear regression. Through the process of comparing the output value and the estimated value, learning of the artificial neural network proceeds in the direction of minimizing it based on the cost function value capable of quantifying it. In this scenario, 'ANSYS Fluent', which is a widely used computational fluid analysis software, was used to extract aerodynamic data through shape design and fluid analysis. By defining preprocessing and input / output of extracted aerodynamic data, learning of neural network. All learning data was separated for learning and validation of artificial neural network, and the accuracy of artificial neural network for the input and output of separated data was verified as a progressive measure for numerical interpolation.
\end{abstract}

Keywords: machine learning, supervised learning, fluid analysis, multi-layer perceptron

\section{Introduction}

The Flush Air Data Sensing (FADS) system, which collects flight data such as flight speed, angle of attack, side-slip angle, and altitude in a high-speed flight such as a space shuttle, or an unmanned combat air vehicle (UCAV) compared to existing pitot tube. It has a buried shape sensor, so it has the advantage of collecting aerodynamic data stably.

As with the pitot tube mounting position, the FADS sensor embedded in the head of the aircraft, would operate unlike the conventional aerodynamic data sensor mechanism, so calibration needs to be performed on the altitude and attitude of the aircraft in order to achieve optimum performance. When estimating the state of the aircraft based on the collected aerodynamic data, it is possible to derive the model equation for the fluid, or to estimate the state of the aircraft by comparing the data conditions based on the accumulated data. However, existing estimation methods were basically a human intuition or logic involved in the verification between all the data, so that there is a certain limit due to the difference between the actual flow and the ideal flow as well as the large load.

Received (May 12, 2018), Review Result (July 21, 2018), Accepted (August 10, 2018)

* Corresponding Author 
This work conducted using a machine learning method based on artificial neural networks, instead of model formula estimation or data analytical approaches. By using the collected data as learning data of artificial neural networks, the real value can be estimated. Since machine learning has its own logic through learning after designating data and structure for specified input and output, high automation can be expected compared with the previous method, as well as the amount of data to be supplied and the size of the structure. It can be expected that the performance will be improved proportionally.

\subsection{Embedded Air Data Sensor}

For estimating the velocity or altitude of the aircraft, the pitot-tube for measuring and collecting the dynamic pressure of the aircraft should be installed in a position that is not influenced by the boundary layer caused by the shape of the aircraft and that is not influenced by the air turbulence. Measurement error can be minimized [1].

But, by using the FADS system, which has a different shape form compared to legacy pitot-tube that is mounted to protrude out of the aircraft body, in order to overcome the limitation of the mounting position, it is possible to measure the stagnation pressure by measuring at the same time. It is also possible to estimate posture such as angle of attack or side- slip angle of an aircraft including speed and altitude information.

\subsection{Numerical Interpolation for Embedded Air Data Sensor}

For the estimation and correction of the atmospheric conditions required for aircraft operation, the establishment of the database for the pressure measured, atmospheric pressure, and the support angle, etc., is required first. The placement of the hole in a FADS system to measure pressure values is located in the air terminal, as in conventional aircraft, and the measured pressure data is in the digital data form [2].

In the previous methods, the flow around the head of the aircraft was assumed to be an ideal potential flow, and a simple model equation was derived or used. The 'Triple Algorithm' can be applied as an estimation and correction model of the atmospheric state, but the relationship between mach number, angle of attack and side-slip angle is nonlinear according to the measured pressure value.

Due to the limitations of the difference, this work estimating method using the artificial neural network technique was used to approximate the real model. Predictions on the distribution of nonlinear data using artificial neural networks can be made similar to actual estimates of nonlinear adaptation through the process of learning from collected data as learning data. In this work, the proponents aimed to estimate numerically the output value of the input value by performing the machine learning based on the MLP structure, which is a representative structure of the artificial neural network.

\section{Model Formula Estimation of Embedded Air Data Sensor}

For the FADS pressure model, the 'Triples' algorithm used in the NASA to space shuttle is a representative example.

Based on the general FPM(Fluid Physics Module) results, the pressure measured on the surface is the solution to the assumption that it is the head of the sphere.

\section{1. 'Triples' Algorithm}

There are various methods of estimating the angle of attack, side-slip angle, and mach number using the pressure values obtained through FPM. The 'Triples' algorithm can be used to estimate the pressure values at three positions in previous FADS system. The pressure estimation formulas based on the euler method, each three positions $(i, j, k)$ are summarized as follows. 


$$
\Gamma_{i k} \cos ^{2} \theta_{j}+\Gamma_{j i} \cos ^{2} \theta_{k}+\Gamma_{k j} \cos ^{2} \theta_{i}=0
$$

And, it is possible to remove $q_{c}, p_{\infty}$ and $\epsilon$ terms from three points by using the equation obtained from the FPM, and reorganize them using.

$$
\Gamma_{i k}=\left(p_{i}-p_{k}\right), \Gamma_{i k}=\left(p_{i}-p_{k}\right), \Gamma_{i k}=\left(p_{i}-p_{k}\right)
$$

By using the Euler method as above equation (2), the relation of each port pressure can be known.

\section{Estimation Based on MLP Neural Network}

In the numerical analysis base on the linear regression, the correction was performed using the machine learning based on the MLP Structure.

\subsection{Artificial Neural Network based Data Processing}

If an artificial neural network technique based on nonlinear data processing is applied, it is possible to estimate the output values (Mach number, angle of attack, etc.,) according to the pressure data measured through the atmospheric sensor.

Computational techniques using neural networks are new computational methods that mimic neurons that are the basis of human brain information processing mechanisms, and have learning functions that generate rules by themselves through data.

It is possible to solve problems that are difficult to apply simple calculation repetition using existing numerical methods, that is, problems due to nonlinear relations without efficient algorithms or optimized equations.

MLP, which is the most general structure of the neural network structure, is based on a single layer perceptron structure. The input vector is classified into a plurality of linear types by taking a series of weights and a bias on the input vectors.

By using multiple single layer perceptrons that can be grouped together and logically grouping them into a plurality of classifications, it is possible to cope with the processing of nonlinear data by having performance characteristics that can be classified into a plurality of criteria as in the XOR problem have.

Based on the single - layer perceptron classification, a neural network learning structure based on learning data can be realized by constructing a network in which propagation occurs in a feed - forward manner by using a plurality of perceptrons acting as input of the next layer.

Here, through the distribution of each layer divided into input / hidden / output layers, the training of the neural network and the weights of the internal nodes were adjusted based on the sample data of the learning.

First, weights and biases were initialized for all nodes in each layer, and then the calculation proceeds in the forward direction with respect to the input.

$$
\left\{\begin{array}{l}
f^{\prime}{ }_{j}\left(\text { net }_{j}\right)\left(d_{j}-o_{j}\right), \text { when } j \text { is output layer } \\
f^{\prime}{ }_{j}\left(\text { net }_{j}\right) \sum_{k} \delta_{k} w_{j k}, \text { when } j \text { is hidden layer }
\end{array}\right.
$$

Using the above equation (3), it is possible to control the degree of learning (= regularization) of the entire neural network by setting the sensitivity $\left(\delta_{k}\right)$ in each layer of the artificial neural network as a hyper-parameter for the entire neural network structure, that is a variable can be arbitrarily adjusted by the user. In the output layer $o_{j}$, and the original result value $d_{j}$ for the input.

In the hidden layer, the weight and sensitivity in the previous layer would be summarized and propagated continuously to the next layer. 
After learning in the forward direction going from the input to the output layer, the learning to the back propagation process can be expressed as follows.

$$
\begin{gathered}
\Delta w_{i j}(t)=\eta \delta_{j} o \\
w_{i j}(t+1)=\alpha \Delta w_{i j}(t-1)+w_{i j}(t)+\Delta w_{i j}(t)
\end{gathered}
$$

For a weight that changes based on the learning rate $(\eta)$ and sensitivity $(\delta)$, the weight change to the next layer is affected by the previous layer, so adjusting the weight for the previous layer in the reverse direction is called back propagation process [4].

In addition, 'dropout' was introduced to minimize the cost function. This method minimizes the loss function by adding noise intentionally to the neural network.

The back propagation process is the same as that of the conventional neural network algorithm, but the forward propagation process is different.

$$
\begin{aligned}
& r_{j} \sim \operatorname{Bernoulli}(\mathrm{p}) \\
& \tilde{y}_{J}=r_{j} \times y_{j} \\
& y_{j+1}=f\left(w_{j+1} \times \tilde{y}_{j}+b_{j+1}\right)
\end{aligned}
$$

Using equation (3), the probability of $\mathrm{p}$ can be applied, which is an independent Bernoulli random variable, to be 1 before entering the activation function $(f)$.

By multiplying 0 or 1 , the neuron output value of the layer is transferred to the next layer or not. Since this series of processes is performed for the entire network, it is similar to the method of sampling a subnetwork on a large network [5].

Here, for more accurate estimation, it is possible to clearly define the input and output data related to the flow around the aircraft, and to conduct future learning through preprocessing of sample data (= learning data) for efficient learning.

\subsection{Feature Selection to Dataset as Pre-Process for Efficient Learning}

The shape of the head of the aircraft was arbitrarily cone-shaped using computational fluid software ('ANSYS Fluent').

For the change of the pressure data condition by each meshes, the mach number of 0.2 to 0.85 (5 kinds), the angle of attack of -4 to 16 degrees ( 11 kinds) and the side slip angle of 0 to 8 degrees ( 5 kinds). The number of combinations was $275(=5 \times 11 \times 5)$, and each condition was extracted as a separate file.

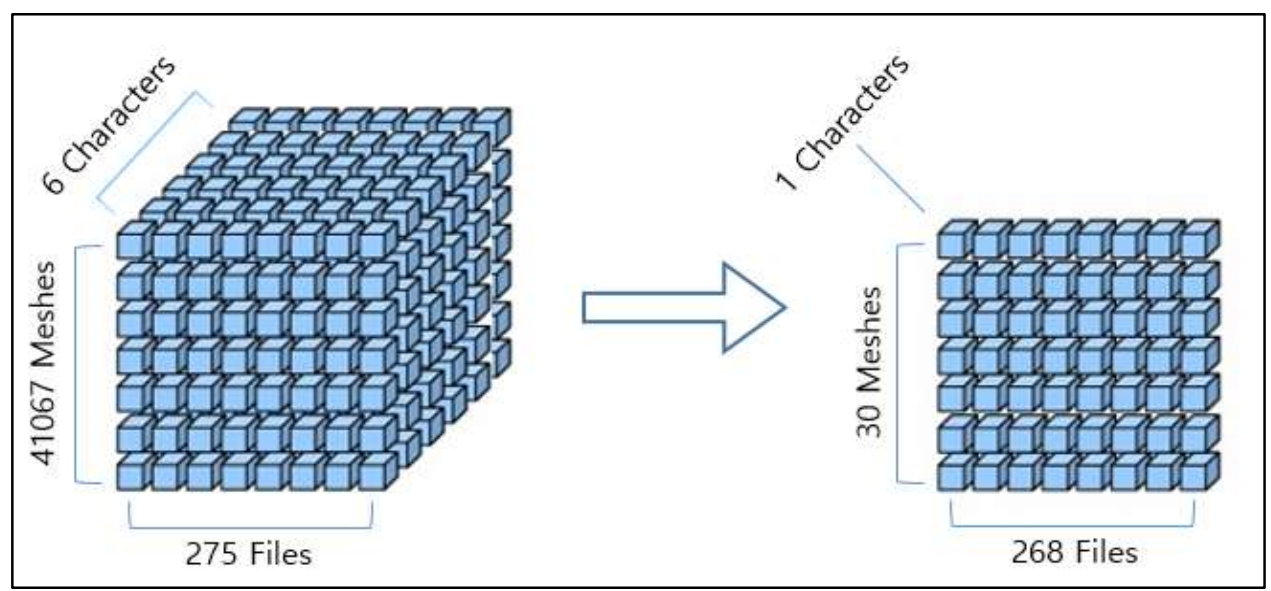

Figure 1. Pre-Process to Dataset 
The number of meshes with respect to the shape of the head of the aircraft in the individual file is 41067, which has a total of six characteristics. However, in order to conduct the actual learning of the machine, it is necessary to pre-process the data for efficient data learning. In this work, the only considered characteristic is the stagnation pressure value among the six characteristics extracted in each individual files.

In addition, a comparison of the simulated aerodynamic data through a 3-D plot shows that files with invalid conditions were excluded. The shape of the data processed through the pre-processing can be shown in Figure 1.

On the other hand, selecting some of the entire meshes can also be used to determine the sensitivity and accuracy of the sensor location and quantity when data is actually collected using the sensor. For this reason, instead of using all the meshes, pre-processing was performed by selecting the points with high sensitivity selectively.

Studying the 40,000 meshes without data pre-processing, the results can be shown following Figure 2.

As shown in Figure 2, the estimation of the actual data was similarly performed, however it can be seen that does not converge below a certain level occurs.

Further, in this case, since a large amount of data flow occurs, a problem arises that a longer time should be required despite the unsatisfactory estimation performance. For this reason, it is necessary to consider efficient learning and computing power of data, and the following structure can be considered for this.

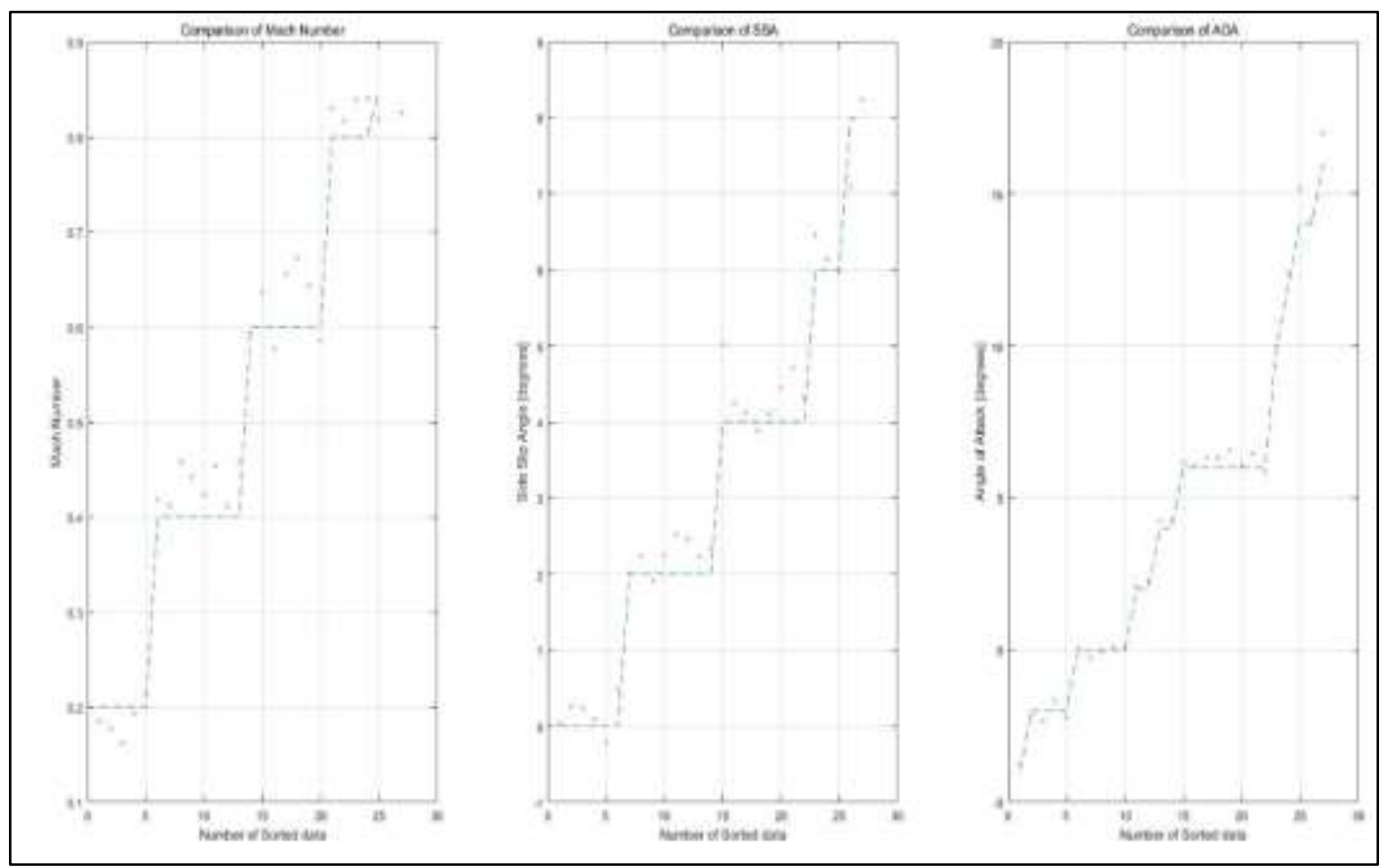

Figure 2. Pre-Processing to Data Set

The characteristics were selected to reduce the number of data and to enable efficient learning. The top 10 features with high scores using characteristic scores according to the statistical based univariate changes were calculated for each Mach number, Side-Slip angle and Angle-of-Attack.

The neural network structure consists of 30 input layers nodes, each 128 first and second hidden layer nodes, and the 3 output layer nodes for train. 


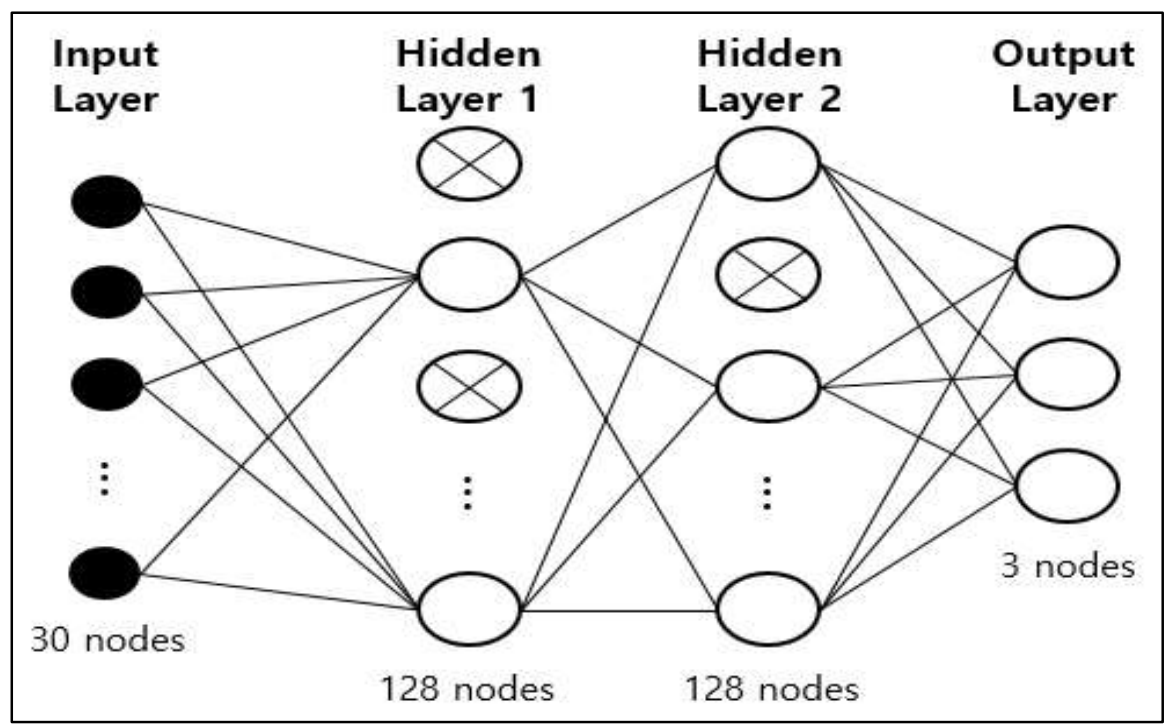

Figure 3. Feed-Forward MLP Structure with Dropout

In order to evaluate the accuracy of trained neural network, about $30 \%$ of the total learning data be separated for validation. However in this work, only $10 \%$ of the data was separated because of relatively small amount of data compared to other big data. It can be judged that the evaluation of data interpolation performance for the trained neural network and the accuracy of the neural network can be evaluated at the same time. And the percentage of nodes that were dropped out is typically around $10 \%$ for linearity, so just $90 \%$ hidden layer nodes were activated for training.

\subsection{Comparison of Neural Network Accuracy by Hyper-Parameter Differences}

The Table 1 Shows the comparison of the variances for each trained neural network after using pre-processed data. Experiments were conducted on the dispersion value of the estimation by a method of confirming the change of the estimation result through the change of the hyper-parameter that the user arbitrarily changed.

Table 1. Comparison of Each Trained Neural Network

\begin{tabular}{|c|c|c|c|c|c|c|c|}
\hline Case & $\begin{array}{c}\text { Learning } \\
\text { Rate }\end{array}$ & $\begin{array}{c}\text { Training } \\
\text { Epochs }\end{array}$ & $\begin{array}{c}\text { Dropout } \\
\text { Rate }\end{array}$ & Batch Size & $\begin{array}{c}\text { Var. of } \\
\text { Mach } \\
\text { Number }\end{array}$ & $\begin{array}{c}\text { Var. of } \\
\text { Side-Slip } \\
\text { Angle }\end{array}$ & $\begin{array}{c}\text { Var. of } \\
\text { Angle-Of- } \\
\text { Attack }\end{array}$ \\
\hline $\mathbf{1}$ & 0.000003 & 80000 & 0.70 & 11 & 0.00911 & 3.05202 & 40.3584 \\
\hline $\mathbf{2}$ & 0.000100 & 100000 & 0.70 & 11 & 0.00056 & 1.53174 & 2.24043 \\
\hline $\mathbf{3}$ & 0.000100 & 100000 & 0.95 & 11 & 0.00202 & 0.06395 & 0.04058 \\
\hline $\mathbf{4}$ & 0.000100 & 150000 & 0.95 & 11 & 0.00036 & 0.03081 & 0.00361 \\
\hline
\end{tabular}

Of course, in fact, the above three cases were considered and trained in various hyperparameter settings, but the results were shown selectively because of the differences.

Also, Table 1 can be visually expressed to the following Figures.

From the left to right, the order of Mach Number, Side-Slip Angle, and Angle-Of-Attack are shown. The original result value is indicated by dotted line and the estimated value is indicated by dot.

From the results shown in the figure below, it can be seen that the hyper-parameter adjustment of the neural network sequentially adjusts it like the actual one. Especially, in case 4 , it can be confirmed that the estimation proceeds almost in the same way as the original result data. 
In general, the higher the learning rate, training epochs, and the dropout rate (the higher number, the fewer the number of inactivated nodes), the better the accuracy.

These results can be considered in various ways. First, even if it is mathematically or theoretically inefficient, the performance improvement can be achieved by continuing the learning by using the computing resources. Second, if the size of the neural network is not large or complicated, it can be seen that the various techniques used in the deep-learning field now have a greater risk than effects.

Finally, it's about the size of the batch. The batch size is usually considered in machinelearning case similar to voice recognition or image processing. If data to be processed at one time would be large, it would be used to reduce the load of computing resources and to focus on efficient learning.

In this work, however, the emphasis was on efficiently adjusting the degree of learning. When put all the data all at once, results were cannot properly derived so the proponents experimentally adjusted our batch size on neural network [6].

This is a phenomenon that can be seen in various fields. The theoretical basis of proper batch size is relatively rare, and it can be seen as a dispute that there is room for further development of machine learning in the future.

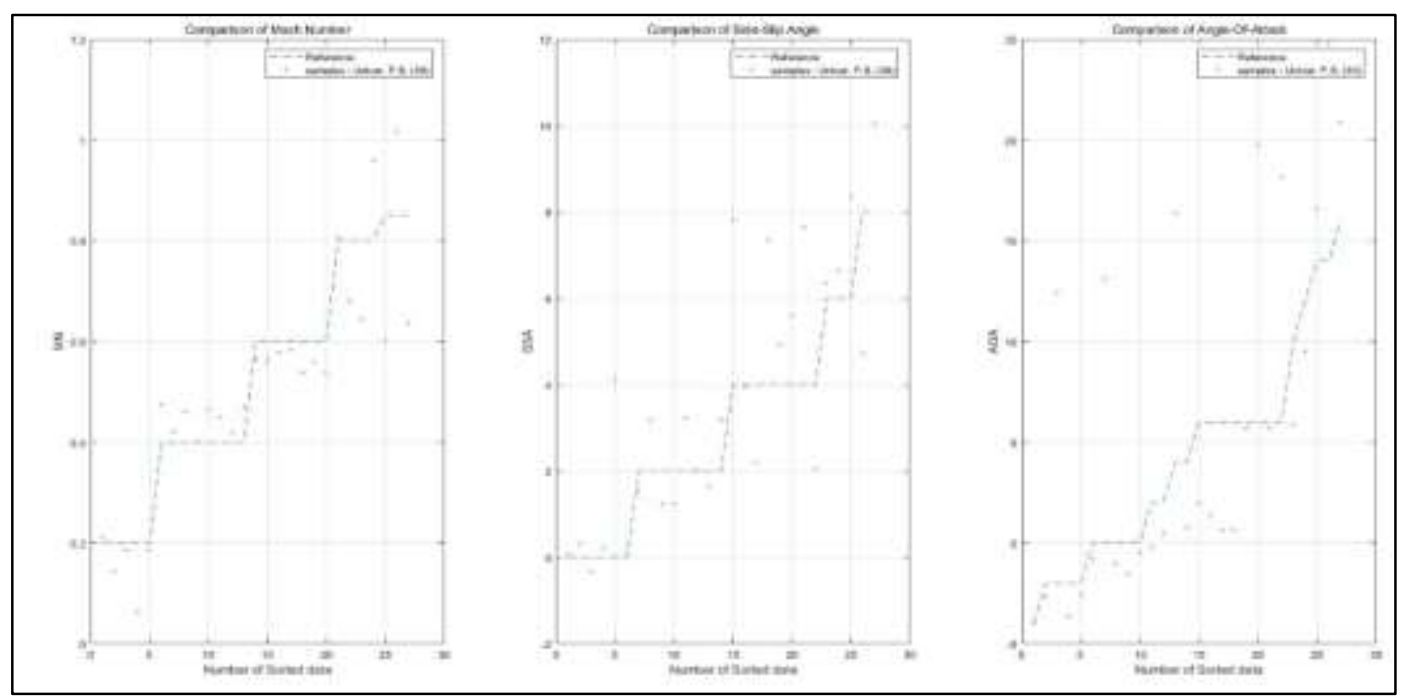

Figure 4. Case 1: Aircraft State Estimation by Neural Network

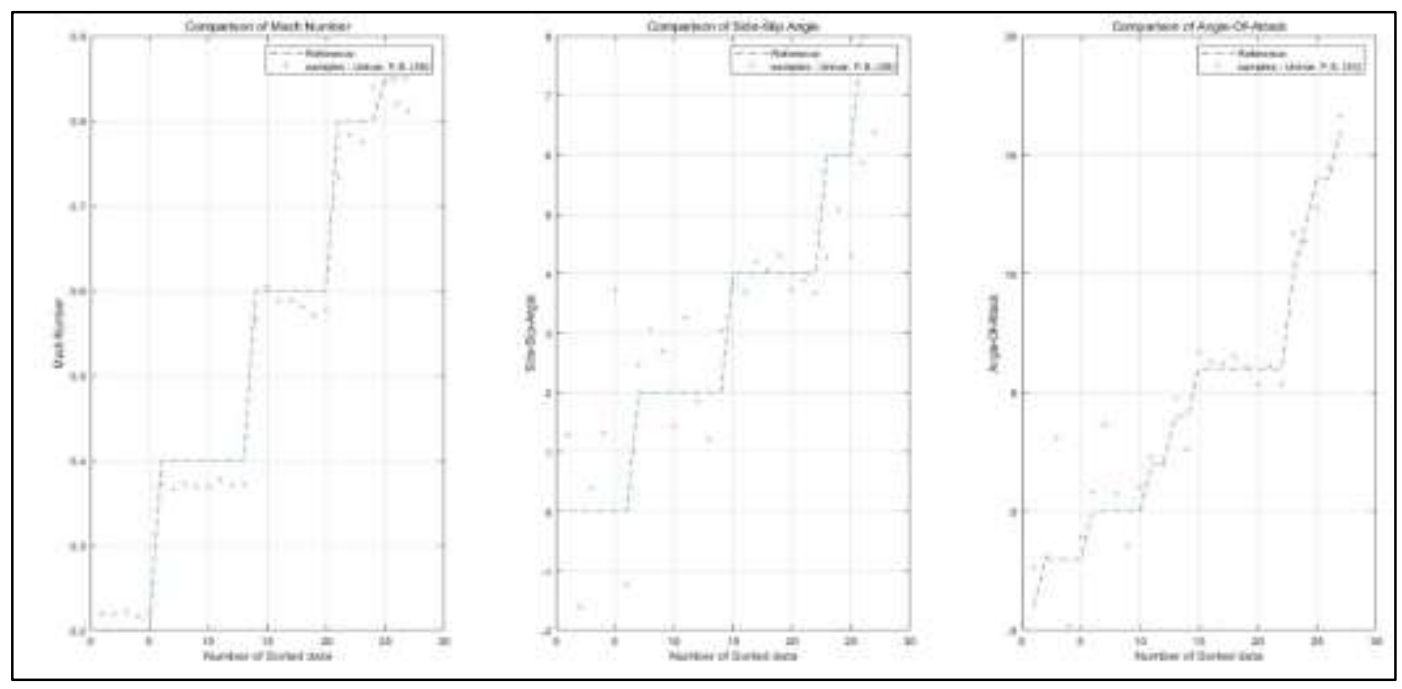

Figure 5. Case 2: Aircraft State Estimation by Neural Network 
For case 1 and case 2, it can be seen that the estimated value does not converge sufficiently, but judging through comparison between case 3 and case 4 where convergence progresses to some extent.

Case 1 has relatively low learning rate compared to other cases, and it can be judged that under fitting occurs because the neural network is not sufficiently learned.

Case 2 has a low dropout rate compared to the converged case, so that the number of inactive nodes is relatively large, which can be regarded as an obstacle to learning.

But, in this case the dropout technique is more advantageous in estimating the mach number better than the side-slip angle or angle of attack.

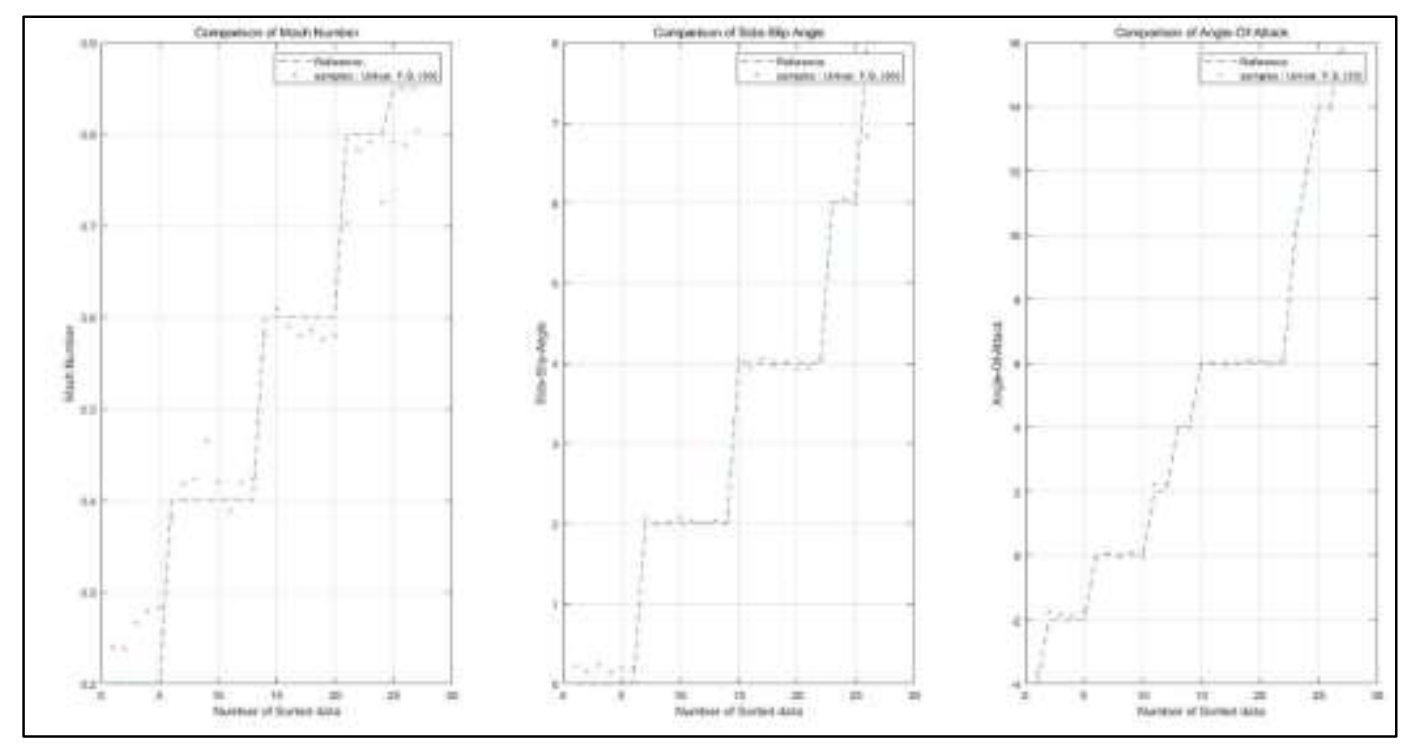

Figure 6. Case 3: Aircraft State Estimation by Neural Network

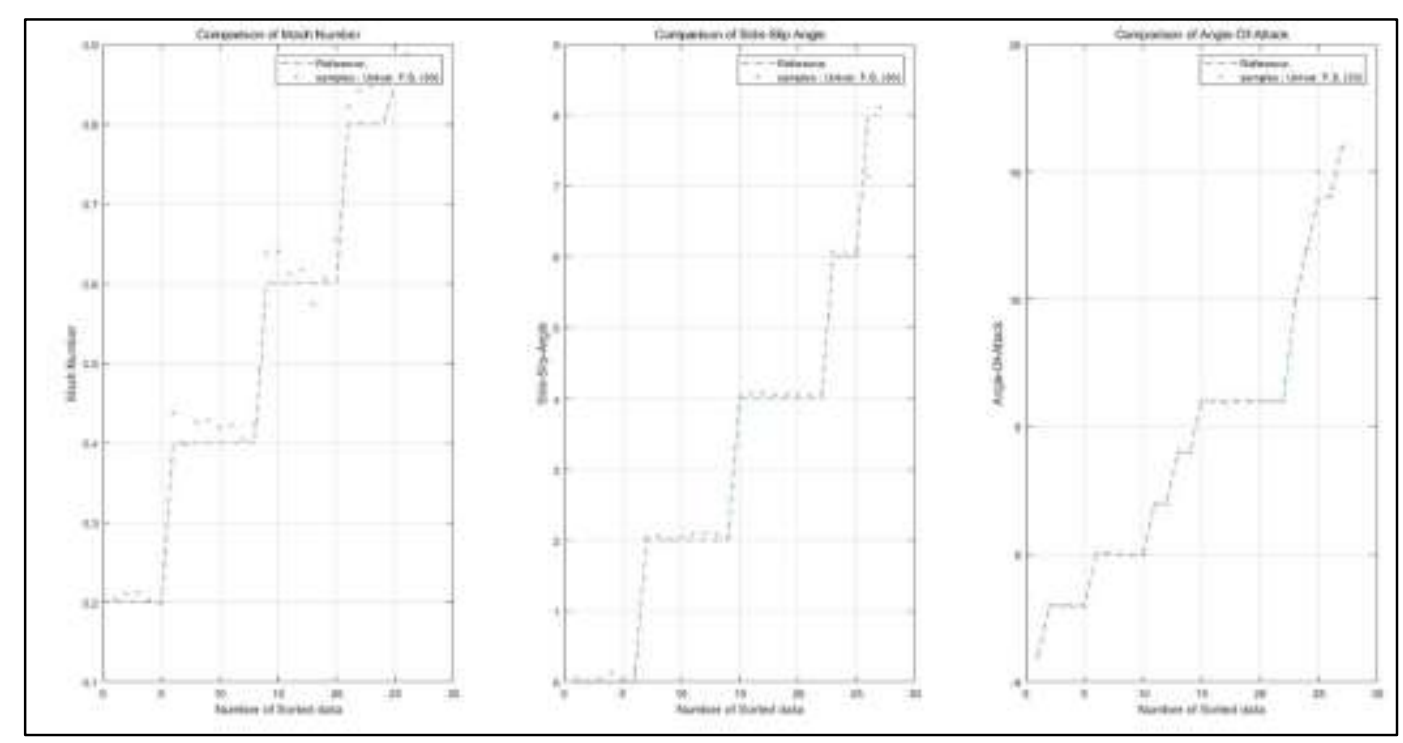

Figure 7. Case 4: Aircraft State Estimation by Neural Network

For three aerodynamic data estimates, the Mach number is relatively inferior to the other estimates of accuracy. It can be considered that the nonlinearity of the neural network structure through the activation function was not properly reflected in the Mach number characteristic linearly with respect to the aircraft pressure. 
In addition, it can be observed that the accuracy decreases as the number of inactive nodes decreases by decreasing the dropout ratio. This is because the neural network structure itself was not large, so it can be considered that the performance loss was larger than dropout data noise removal.

\section{Conclusion}

In this paper, based on the aerodynamic data collected through the simulation, a study on whether it is possible to estimate similarity to the actual case of machine learning was conducted. In order to make the numerical interpolation, some of the whole data was separated and used for the validation, and the other data was used to the learning data for numerical interpolation.

Because of the characteristics of the neural network structure by activation function, based on the nonlinearity, stable learning is possible for side-slip angle and angle-of-attack which contains nonlinearity. However, for the input and output data, it can be observed that the accuracy of learning was somewhat lower in the case of mach numbers with a linear relationship.

In order to stabilize the numerical estimation and interpolation using machine learning methods in the future, it can be considered that stable data processing can be performed by considering a separate neural network structure considering linearity.

Also, in the process of selecting a specific mesh in the entire mesh, it can be considered that a more efficient method can be applied if the importance was determined as the machine learning itself by using the wrapper method or the embedding method in addition to the filter method used in the present invention.

\section{Acknowledgments}

This paper is a revised and expanded version of a paper entitled [Numerical Interpolation of Aircraft Aerodynamic Data through Regression Approach on MLP Network] presented at $\left[10^{\text {th }}\right.$ International Conference on $\mathrm{u}-$ and e- Service, Science and Technology (UNESST2018), Hilton Hanoi Opera, Hanoi, Vietnam, July 12-14].

This work was supported by a grant from Hanseo University in 2018.

This work was supported by the Korea Institute for Advencement of Technology(KIAT) grant funded by the Korean government(Motie : Ministry of Trade, Industry \& Energy) (No. N0002431).

\section{References}

[1] J. C. Choi, K. G. Lee and C. W. Park, "Development of the Pitot-Tube to Measure Flight Altitude and Velocity", Journal of Korean Society of Propulsion Engineers, vol. 4, (2001), pp. 19-24.

[2] Y. Liu, D. Xiao and Y. Lu, "Comparative Study on a Solving Model and Algorithm for a Flush Air Data Sensing System”, Open Access Journal - Sensors, vol. 14, (2014), pp. 9210-9226.

[3] B. R. Cobleigh, S. A. Whitmore and E. A. Haering, Jr., "Flush Airdata Sensing(FADS) System Calibration Procedures and Results for Blunt Forebodies", NASA/TP-1999-209012, (1999).

[4] F. Vargas, D. Lettnin, M. C. F. de Castro and M. Macarthy, "Electrocardiogram pattern recognition by means of MLP network and PCA: a case study on equal amount of input signal types", Proceedings of VII Brazilian Symposium on Neural Networks, Pernambuco, Brazil, (2002) November 11-14.

[5] N. Srivastava, G. Hinton, A. Krizhevsky, I. Sutskever and R. Salakhutdinov, "Dropout: A Simple Way to Prevent Neural Networks from Overfitting”, Journal of Machine Learning Research, vol. 15, (2014), pp. 1929-1958.

[6] M. Li, T. Zhang, Y. Chen and A. J. Smola, "Efficient mini-batch training for stochastic optimization", Proceedings of the 20th ACM SIGKDD International Conference on Knowledge Discovery and Data Mining, New York, New York, USA, (2014) August 24-27. 

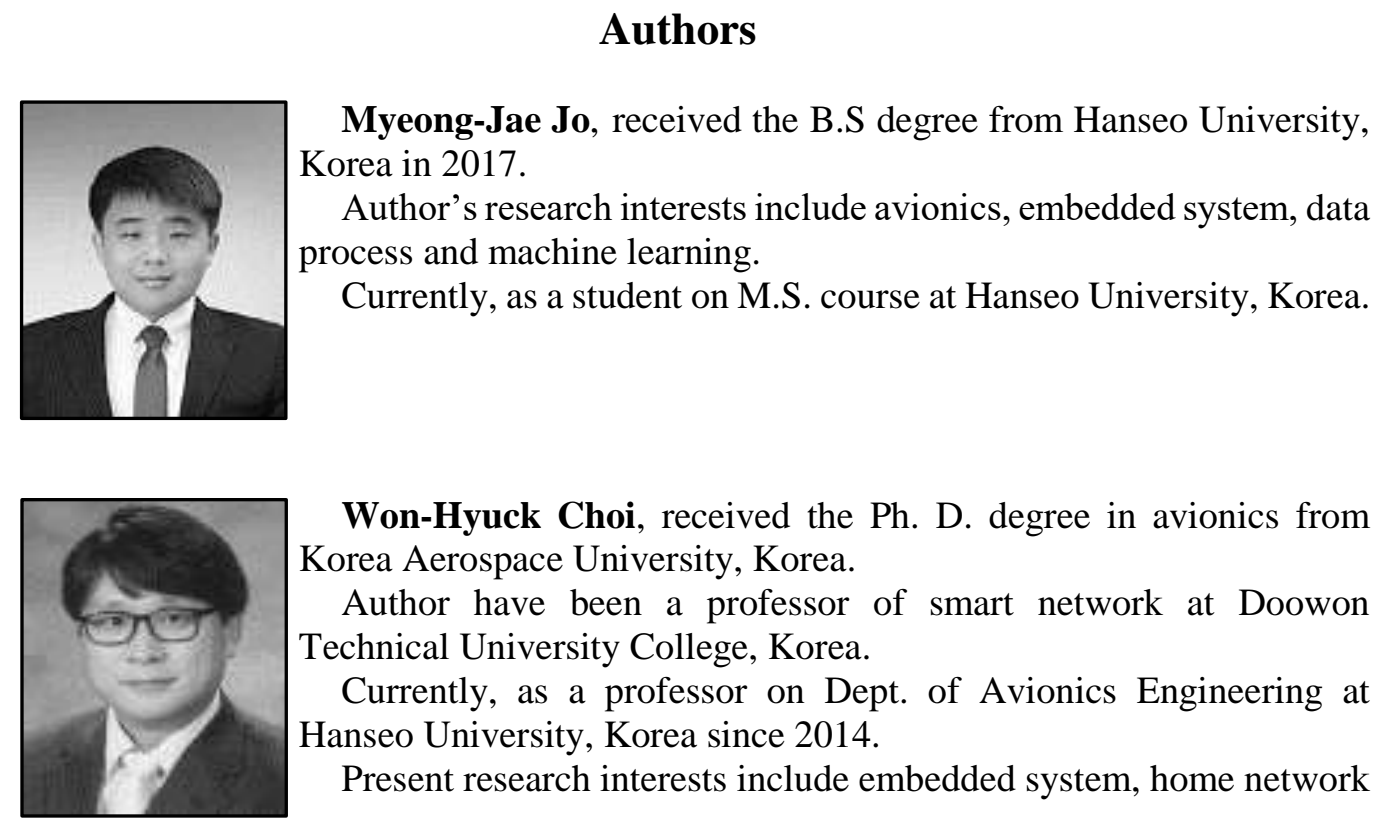

Won-Hyuck Choi, received the $\mathrm{Ph}$. D. degree in avionics from Korea Aerospace University, Korea.

Author have been a professor of smart network at Doowon Technical University College, Korea.

Currently, as a professor on Dept. of Avionics Engineering at Hanseo University, Korea since 2014.

Present research interests include embedded system, home network

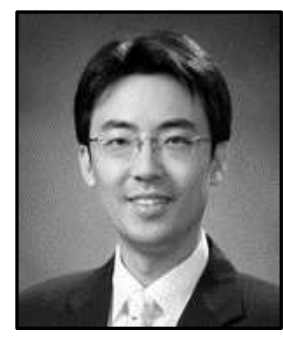

Min-Seok Jie (Corresponding Author), received the Ph. D. degree in avionics from Korea Aerospace University, Korea.

Author was a Researcher of Intelligent Robot Reseach Center at Korea Institute of Science and Technology from 2007 to 2008.

And, as a visiting professor at Purdue University, US from 2015 to 2016.

Currently, as a professor on Dept. of Avionics Engineering at Hanseo University, Korea since 2008.

Present research interests include robust control, robot, vision based control and UAV. 